\title{
Do Halo Red Giant Stars Contribute to the High-Velocity Gas Falling onto the Milky Way Disk?
}

\author{
Klaas S. de Boer \\ Sternwarte der Universität Bonn, D-53121 Bonn, Germany
}

Abstract. The amount of gas shed by red giant stars in the halo is assessed to find its contribution to the long known infall of high- $b$ gas. The infall rate due to $\mathrm{RG}$ star mass loss equals $3 \cdot 10^{-6} \mathrm{M}_{\odot} \mathrm{kpc}^{-1} \mathrm{y}^{-1}$.

\section{Introduction}

The detection of complexes of neutral hydrogen gas having their velocity directed toward the Milky Way disk showed that gas clouds are falling onto the Milky Way. It was speculated that the gas might come from intergalactic space (Oort 1966), in which case it should be almost free from metals. The discovery of this influx of possibly pristine matter was embraced by modellers of the evolution of the Milky Way. The "closed box" evolution models predicted much more metal poor G-dwarfs than actually are known to exist today (Audouze \& Tinsley 1976; for comments Pagel 1997). Since that time the amount of infall needed in the evolution models was taken either as free parameter or set equal to the amount of infall as can be estimated from the observed halo high-velocity clouds (HVCs).

Halo gas falling toward the disk of the Milky Way has long been thought to come from a galactic fountain like flow or perhaps falling in from intergalactic space. Since red giant stars lose mass and since there are many in the halo, the amount of gas shed by those older halo red giants is estimated.

\section{Halo RG stars}

The red giants (RGs) evolve and lose mass and end up as horizontal-branch (HB) stars. HB stars have a He core of $\simeq 0.5 \mathrm{M}_{\odot}$, surrounded by a hydrogen shell of between 0.05 and $0.4 \mathrm{M}_{\odot}$. For the old halo RGs $\dot{M}_{\text {one } \mathrm{RG}} \simeq 0.3 \mathrm{M}_{\odot}$.

To calulate the total mass lost by RG stars, the spatial distribution of RGs in the halo, and the total number of RGs has to be known. However, the RG star distribution is essentially unknown and therefore must (and can!) be derived in an indirect manner. The sdB stars serve the purpose. They are a subclass of HB stars and HB stars have RG stars as progenitors.

The $z$-distribution of field sdB stars has been studied by de Boer et. al (1997) and Altmann et. al (2003). They find a disk component with an exponential distribution with scale height of $h_{\text {disk sdB }} \simeq 0.9 \mathrm{kpc}$ and a halo component characterized by a scale height of $h_{\text {halo } \mathrm{sdB}} \simeq 7 \mathrm{kpc}$. The midplane density $n(0)$ of sdB stars is available from Downes (1986) at $n(0)_{\text {disk } \mathrm{sdB}} \simeq 2 \cdot 10^{-6} \mathrm{pc}^{-3}$. 


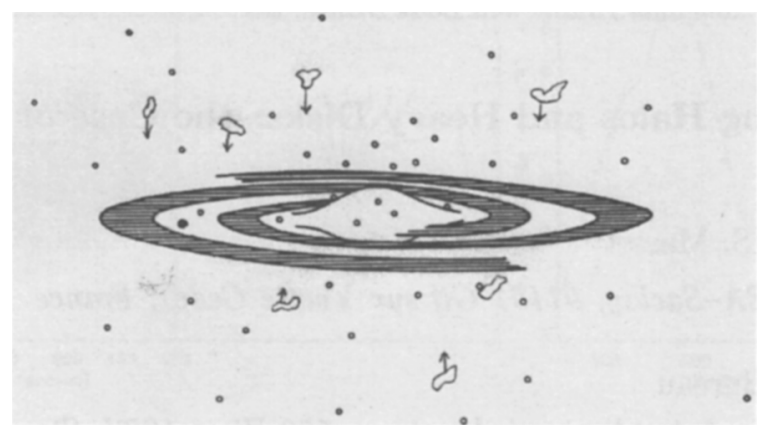

Figure 1. The Milky Way contains globular clusters and clouds of neutral infalling gas. Some clouds are due to a galactic fountain like flow, a small fraction perhaps from intergalactic space, while a good portion is due to mass lost by the RG stars of the Milky Way halo

After integration over the exponential distribution function between $z$ of 1 and $20 \mathrm{kpc}$ the total number of sdB stars per column $N_{\mathrm{sdB}}$ is found.

Furthermore, the ratio of all $\mathrm{HB}$ stars to sdB stars has to be estimated. The sdB stars have $30,000>T_{\text {eff }}>20,000 \mathrm{~K}$, the HBB and HBA stars, the RR Lyrae, and the RHB stars, are cooler. The ratio of the total number of HB stars in the halo to the $\mathrm{sdB}$ stars is estimated to be $n_{\text {all } \mathrm{HB}} / n_{\mathrm{sdB}} \simeq 8 \pm 4$.

\section{Total mass loss rate}

The average mass loss rate of an RG star is found from the assumption of a stationary halo in which HB stars have a phase life of $\simeq 10^{8} \mathrm{y}$. The total halo RG mass-loss rate requires all parameters and is found to be

$$
\dot{M}_{\text {halo RG }}=N_{\text {sdB }} \times n_{\text {all } \mathrm{HB}} / n_{\mathrm{sdB}} \times \dot{M}_{\text {one RG }} \quad \mathrm{M}_{\odot} \mathrm{y}^{-1} \text {. }
$$

Inserting appropriate values leads to $\dot{M}_{\text {halo } \mathrm{RG}}=2.5 \cdot 10^{-6} \mathrm{M}_{\odot} \mathrm{kpc}^{-2} \mathrm{y}^{-1}$. Adding the mass lost by globular cluster RG stars (de Boer 1985) the grand total mass loss as infall on the MW disk is $\dot{M}_{\text {halo }} \mathrm{RG}=3.0 \cdot 10^{-6} \mathrm{M}_{\odot} \mathrm{kpc}^{-2} \mathrm{y}^{-1}$. A full paper will be submitted to A\&A.

\section{References}

Altmann, M., Edelmann, H., \& de Boer, K.S. 2003, A\&A, submitted Audouze, J., \& Tinsley, B.M. 1976, ARA\&A, 12, 43

de Boer, K.S. 1985, A\&A 142, 321

de Boer, K.S., Aguilar Sánchez, Y., Altmann, M., Geffert, M., Odenkirchen, M., Schmidt, J.H.K., \& Colin, J. 1997, A\&A 327, 587

Downes, R.A. 1986, ApJS 61, 569

Oort, J.H. 1966, Bull. Astron. Inst. Neth., 18, 421

Pagel, B.E.J. 1997, 'Nucleosynthesis and chemical evolution of galaxies', Cambridge Univ. press 